\title{
CRITERIOS DE INTERVENCIÓN EN FORJADOS CON VIGUETAS DE CEMENTO ALUMINOSO
}

\author{
(UPGRADING CRITERIA FOR SLABS WITH BEAMS MADE OF HIGH ALUMINA CEMENT)
}

\author{
Xavier Casanovas y Joan Ramón Rosell \\ Col. legi d'Aparelladors i Arquitectes Tècnics de Barcelona \\ Escola Universitària Politècnica de Barcelona \\ ESPAÑA
}

Fecha de recepción: 11-XI-92

\begin{abstract}
RESUMEN
El artículo recoge la problemática referida a la seguridad estructural de 900.000 viviendas construidas con cemento aluminoso en el Estado Español, entre 1950 y 1970.

Se plantean las dificultades que representa el actuar en un parque tan extenso y con sus ocupantes en el interior, proponiéndose tres grupos de soluciones: 1.-Aceptar el comportamiento actual en base a una diagnosis fiable y con aplicación de medidas de prevención, seguimiento en servicio $y$ limitaciones de uso; 2.-Reparaciones y refuerzos, mejorando la capacidad portante o reduciendo las solicitaciones; 3.-Substitución de los elementos en mal estado, fisica o funcionalmente, presentándose diversas propuestas actualmente industrializadas que optimizan y facilitan los trabajos de este tipo, aplicando diferentes técnicas y materiales.
\end{abstract}

\section{SUMMARY}

This article deals with the problems concerning structural security of 900,000 houses built with high alumina cement in Spain between 1950 and 1970 .

Three groups of solutions are suggested in order to make this difficut mission possible - carry out the intervention in such a large estate with the occupants inside. 1.-Accept the present performance with the help of a reiable analysis, applying the prevention measures, service control and limited use; 2.-Repair and reinforcements improving the bearing capacity or decreasing the acting loads; 3.-Replacing the members in poor physical or functional conditions; there are various proposals for application of different techniques and materials (whose production is already industrialized) which would optimize and facilitate such interventions.

\section{ASPECTOS PREVIOS}

Afrontar los problemas que puede presentar un techo construido con viguetas de hormigón, si lo planteamos de una forma individualizada, no supone mayores dificultades de las que tradicionalmente han venido comportando la reparación de los techos construidos con vigas de madera. La filosofía intrínseca de cualquier intervención en este sentido, se apoyará en principios muy similares.
La problemática generada por el uso del cemento aluminoso en la fabricación de viguetas, como queda ampliamente definida en otros artículos del presente número, excede los aspectos puramente técnicos, para adquirir una dimensión mucho más extensa que abarca una problemática social y económica de grandes dimensiones, llevando el problema por la vía de los planteamientos de tipo político. 
Informes de la Construcción, Vol. 44 n. ${ }^{\circ}$ 422, noviembre/diciembre, 1992

Dar respuesta a la inquietud ciudadana generada por la degradación que puede derivarse de la utilización del cemento aluminoso en aproximadamente 900.000 viviendas repartidas de forma desigual por todo el territorio del Estado español, requiere la creación de un marco unitario en el que participen: las diferentes administraciones competentes en la materia, los técnicos que deben realizar los trabajos de detección, diagnóstico y reparación, así como los agentes sociales representados por asociaciones de vecinos $\mathrm{u}$ otros organismos.

Este marco unitario, debería avanzar en el conocimiento técnico del problema, plantear unos criterios contrastados en todas las fases de la actuación, delimitar las responsabilidades de los actuantes y establecer unas medidas legislativas en los ámbitos económico, jurídico y social. Herramientas todas ellas imprescindibles para regular el necesario esfuerzo.

En este sentido, resulta interesante destacar que el problema generado por el cemento aluminoso ha sacado a la luz el estado de conservación y las condiciones de seguridad de un amplio parque edificado, construido con unos medios y unas "calidades" realmente bajas que sirvieron, en una época, para resolver una necesidad de alojamiento masivo con unos recursos económicos limitados. Hoy parece claro que no podemos circunscribir la problemática tan sólo a los forjados con cemento aluminoso, ya que los estudios realizados hasta hoy ponen de manifiesto degradaciones también graves en forjados con cemento portland, así como en otros elementos constructivos que obligan a reflexionar respecto a la posible vida útil de estos edificios con simples refuerzos de forjados y en los costes reales de reparaciones integrales.

Llegados a este punto, consideramos necesario advertir que los criterios estrictamente técnicos que a continuación se exponen pueden resultar, en muchos casos, tan sólo una visión parcial de una realidad a menudo compleja, difícil y cargada de matices. Las actuaciones politicas son la única vía efectiva para la resolución del problema en su verdadera magnitud. Resulta imprescindible una Política de Vivienda que contemple la substitución de edificios deficientes, en base a estudios específicos referidos al estado del parque edificado, que permitan programar las actuaciones de renovación sistemática a partir de las urgencias por falta de seguridad que ya se están planteando y que sin duda se incremen-

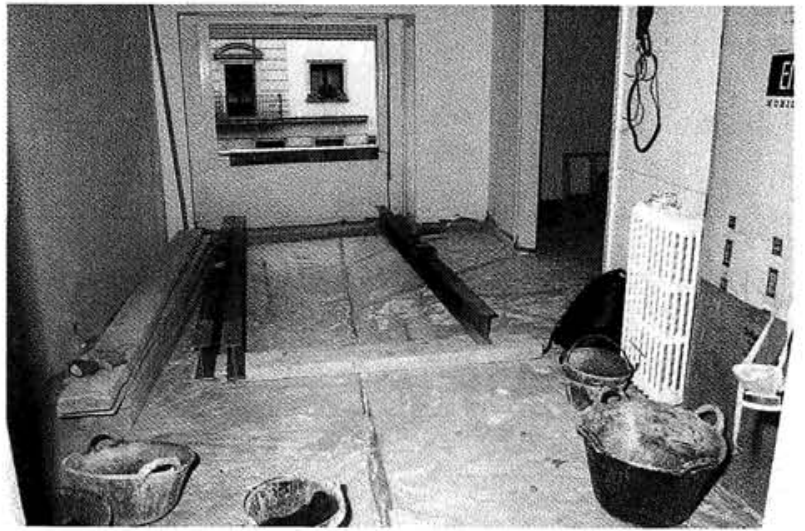

Foto 1.-La protección del pavimento, paredes, ventanas, etc. se hace absolutamente necesaria para no causar desperfectos en las viviendas.

tarán en los próximos años, hasta la renovación total de este parque a unos 20 ó 30 años vista.

\section{DIFICULTADES PARA ACTUAR}

Como se ha comentado, nos encontramos ante un caso de características singulares, mediatizado por una serie de factores que comportan dificultades específicas en las actuaciones que es necesario tener presentes al plantear unos criterios de intervención.

\section{Dificultades no técnicas}

En este apartado comentaremos algunos de los aspectos que más frecuentemente se nos plantean y que hay que tener en cuenta a priori, a pesar de que no requieren una respuesta técnica, como son:

- Por lo general estas actuaciones se realizan en edificios ocupados por sus habitantes, lo que exige una programación de los trabajos muy esmerada para causar las menores molestias posibles (foto 1).

- La mayoría de edificios afectados están en régimen de propiedad horizontal con intereses opuestos entre los diferentes vecinos, resultando difíciles los acuerdos por unanimidad y las actuaciones integrales.

- Son muchos los casos en los que los vecinos afectados no tienen la disponibilidad económica para hacer frente a los gastos que se van a generar y los sistemas de financiación, hasta hoy, no son lo ágiles ni flexibles que la situación requiere. 


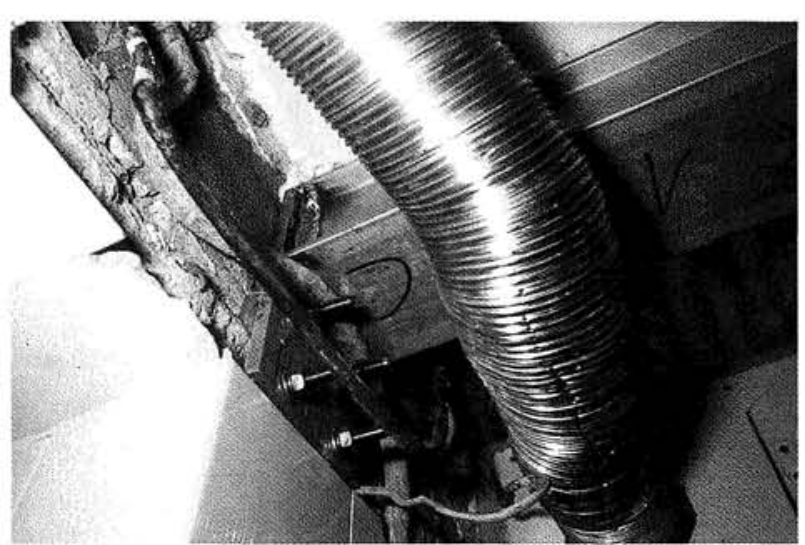

Foto 2.-La aparición de instalaciones de todo tipo en los puntos más inesperados causa dificultades al desarrollo de los trabajos y requiere su adaptación a la nueva situación.

- No resulta fácil para los técnicos afrontar este tipo de actuación sin tener delimitadas con claridad sus responsabilidades respecto a lo que se considere necesario reforzar o conservar.

\section{Dificultades técnicas}

En este apartado comentaremos algunas de las dificultades técnicas que presentan estas actuaciones de forma genérica y que hay que tener presentes en el planteamiento inicial, como son:

- Muy a menudo se presentan dificultades en la visualización de los elementos a inspeccionar, haciendo que operaciones simples adquieran una gran complejidad.

- Hay que conocer las características constructivas y de los materiales que componen el edificio, para aplicar, en cada caso, la solución idónea que contemple los parámetros de seguridad, durabilidad y coste.

- La realización de las pruebas necesarias para poder emitir un diagnóstico representativo fiable implica un gran trastorno para los usuarios y un coste importante en tanto no se articulen métodos y criterios de actuación en este campo de fácil aplicación.

- La existencia de una altura libre interior de las viviendas en el límite de la habitabilidad nos introduce en un campo de difícil solución.

- La existencia de tabiquería de distribución dificulta la accesibilidad de los materiales y los propios trabajos de reparación.
- La existencia de instalaciones empotradas o vistas y los diferentes acabados, requieren trabajar extremando los cuidados o aceptar costos suplementarios importantes de restitución al estado inicial (foto 2).

- La obligatoriedad o no de ajustarse a la normativa actual, no está suficientemente clara y en función de nuestra interpretación podemos variar absolutamente la solución a adoptar.

\section{GRUPOS DE SOLUCIONES}

Ya desde una visión estrictamente técnica y centrados en la actuación en los forjados, agrupamos las posibles soluciones en tres grupos que consideramos representativos de las que actualmente se están aplicando, no incluyendo el derribo del edificio como una alternativa, ya que consideramos que el mal estado de los forjados no justifica esta actuación, si no va a compañado de disfunciones importantes en otros elementos constructivos.

\section{COMPORTAMIENTO ACTUAL ACEPTABLE}

Está demostrado que el cemento aluminoso es un material con buenas prestaciones que requiere unos cuidados en la elaboración de hormigones y en su puesta en obra muy determinados. En general, en la industria de la construcción no existía, ni existe, una cultura tan estricta respecto al hormigón, por lo tanto es lógico encontrar hormigones porosos y con pérdidas de resistencia importantes respecto a la resistencia inicial.

Si bien los hormigones de cemento portland también presentan problemas, son porcentualmente muchos más los problemas de los de cemento aluminoso (en relación 4 a 1).

Diagnosticado el comportamiento actual, si éste resulta aceptable, mantener el edificio en servicio durante un período de tiempo significativo y en unas condiciones de seguridad suficientes parece la opción más razonable y económica.

En este sentido planteamos tres líneas de acción complementarias que pueden contribuir a la conservación en servicio de los edificios por un período de tiempo razonable y en unas condiciones de seguridad correctas. 


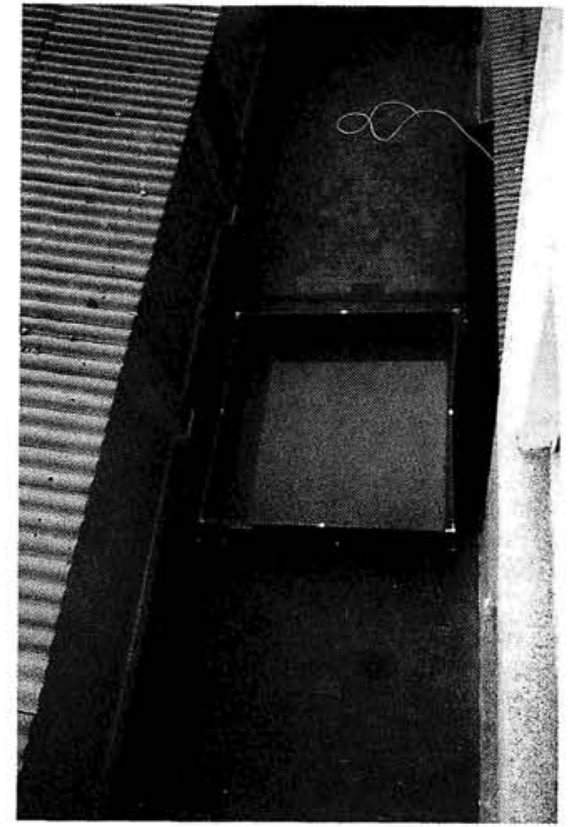

Foto 3.-Las sobrecargas a que se someten muchos forjados superan en muchos casos las previsiones de cálculo, sin considerar el posible deterioro de los elementos portantes.

\section{Limitaciones y recomendaciones de uso}

Como medida de precaución resulta recomendable en estos edificios no incrementar las cargas actuales y en la medida de lo posible, reducirlas, eliminando los elementos excesivamente pesados y evitando las concentraciones temporales de carga.

La normativa de cálculo vigente en los años 50 y 60 era sensiblemente diferente a la actual, adoptándose sobrecargas de uso inferiores. El uso de los edificios también ha ido variando a lo largo de los años y hoy disponemos de elementos mucho más pesados en las viviendas (foto 3 ).

Como resultado del recálculo hay que fijar una sobrecarga admisible cuantificada, con los coeficientes de seguridad apropiados y hay que plantear este dato como límite de uso para los vecinos.

\section{Prevención y protección}

La mayoría de las viguetas de hormigón de cemento aluminoso fabricadas en los años 50 a 70 constituyen, hoy en día, un medio poroso y carbonatado que no garantiza la protección física ni química frente a la corrosión de las armaduras.
Así pues, si su comportamiento actual es aceptable y no se incrementan las sobrecargas de uso, el posible fallo futuro se producirá como consecuencia de la pédida de capacidad portante de la vigueta, por corrosión de su armadura, especialmente grave en los casos de homigón precomprimido.

En este sentido, las medidas preventivas y de protección a adoptar, consistirán básicamente en evitar la presencia de humedades en el edificio en general y en las viguetas muy especialmente.

Ventilación adecuada, substitución preventiva de conducciones envejecidas de suministro de agua y desagües, reparación inmediata de fugas de agua accidentales y de goteras, impermeabilización de paramentos exteriores, mantenimiento en general, siempre serán aconsejables a corto y medio plazo.

La impermeabilización directa de las viguetas o de los forjados presenta muchas dificultades de aplicación. En algunos casos esta impermeabilización puede permitir localmente entradas de humedad y dificultar posteriormente su evaporación, actuando, por tanto, como un factor acelerador de la degradación.

\section{Seguimiento en servicio}

Las medidas comentadas contribuirán a mejorar el uso y la durabilidad de los edificios, si bien para garantizar la seguridad será necesario realizar inspecciones periódicas que permitan observar el comportamiento, en el tiempo, de los forjados afectados.

Para que esta operación resulte más fácil, hay que prever en el período de diagnosis dejar unas zonas registrables o incluso instalar un sistema de monitorización que permitirá un control más continuado de deformaciones o humedades excesivas.

\section{REPARACIÓN Y REFUERZO}

Los problemas inmediatos que se presentan en los forjados, en muchos casos, no afectan a su totalidad, ni tampoco a las viguetas en toda su longitud. Esto nos permite plantearnos un tipo de actuación tendente a reparaciones puntuales de los defectos del elemento o de la parte deteriorada. 
En este sentido planteamos tres actuaciones no excluyentes que siempre llevarán implícitas las precauciones de limitación de uso y seguimiento en servicio expuestas en el apartado anterior.

\section{Reconstrucción de la geometría}

Estas reparaciones puntuales serán apropiadas en los casos en que sea necesario recubrir la armadura que esté desprotegida como consecuencia de lesiones en el hormigón (foto 4). Tan sólo será aconsejable en aquellos casos en los que la armadura se presente en buen estado y con sección suficiente para absorber los esfuerzos previstos. Esta reconstrucción es en general inviable en casos de hormigón precomprimido.

La técnica de intervención es la tradicional de reparación de hormigones con problemas de pérdida de sección, prestando especial atención a la elección del mortero de reparación, así como al posible material de puente de unión.

\section{Mejora de la capacidad portante}

Este tipo de refuerzo se centra fundamentalmente en incrementar la capacidad de trabajo del elemento a flexión y a cortante mediante la fijación de un suplemento de acero con morteros de resinas.

En estos casos se debe considerar el comportamiento al fuego de las resinas, la compatibilidad de los materiales, sus coeficientes de dilatación y otros factores específicos de estas técnicas.

Es importante considerar que, frente a un hormigón de baja resistencia, un incremento excesivo de la cuantía mecánica de tracción puede aumentar el riesgo de rotura frágil por agotamiento de la zona comprimida.

Otra alternativa de mejora de la capacidad portante del forjado, consiste en incrementar su rigidez y su monolitismo en base a la formación de una capa de compresión superior convenientemente conectada, o bien actuando en toda la superficie inferior del forjado, a partir de un gunitado armado u otro sistema de rigidización.

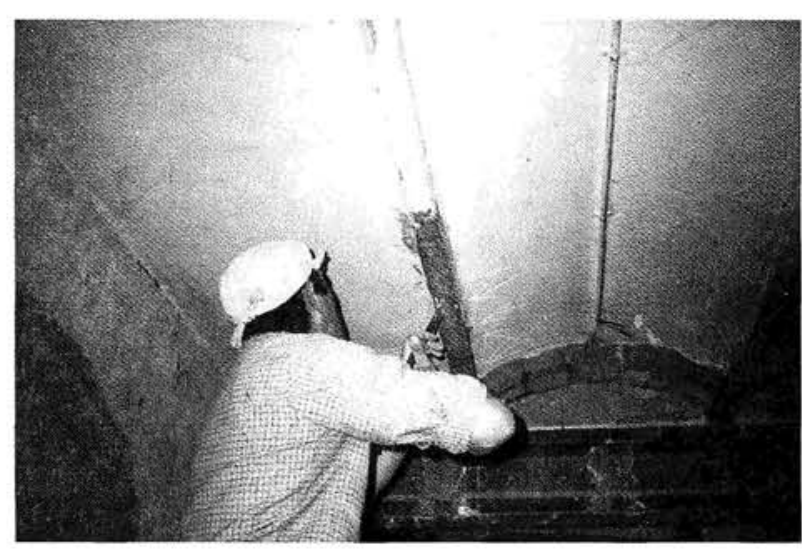

Foto 4.-Reparaciones puntuales como ésta se pueden hacer cuando los problemas están muy localizados y con viguetas armadas.

Este tipo de refuerzos será aplicable en aquellos casos en los que se decida actuar en toda la superficie, pero en general resultan poco viables.

\section{Reducción de las solicitaciones}

En este grupo consideramos aquellas soluciones tendentes a reducir las solicitaciones en las zonas más comprometidas del forjado y que han venido usándose tradicionalmente en todo los tipos de forjado como sistema de refuerzo y sencillo y rápido, resultando más idóneo para forjados de madera y acero y más problemático en los casos de hormigón armado.

Esta reducción de solicitaciones, a menudo comporta una modificación notable del estado tensional de diferentes componentes estructurales del edificio y una alteración del sistema de descenso de cargas.

Para reducir solicitaciones hemos de variar los diagramas de momentos y cortantes que soportan las viguetas. Esta variación la podemos conseguir utilizando tornapuntas en los extremos de las viguetas o vigas parederas, reduciendo las luces libres en base a colocar de forma transversal vigas parteluz, etc.

La posibilidad de recuperar parte de la flecha actual, puede condicionar la entrada en carga más o menos completa del refuerzo, convirtiéndolo en un refuerzo inicialmente activo. Esta es una operación delicada si se realiza sin un análisis previo de la sección.

http://informesdelaconstruccion.revistas.csic.es 


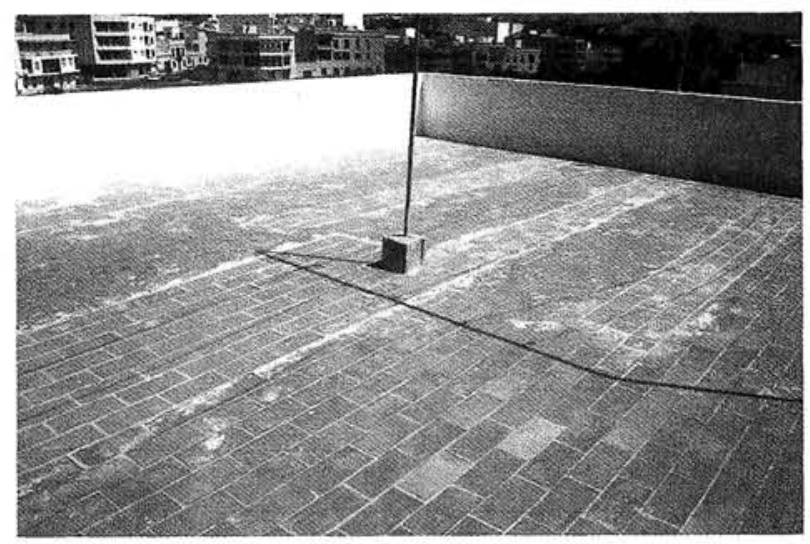

Foto 5.-El caso de las cubiertas, es uno de los que puede aconsejar la substitución física, dada la facilidad de trabajo que comporta.

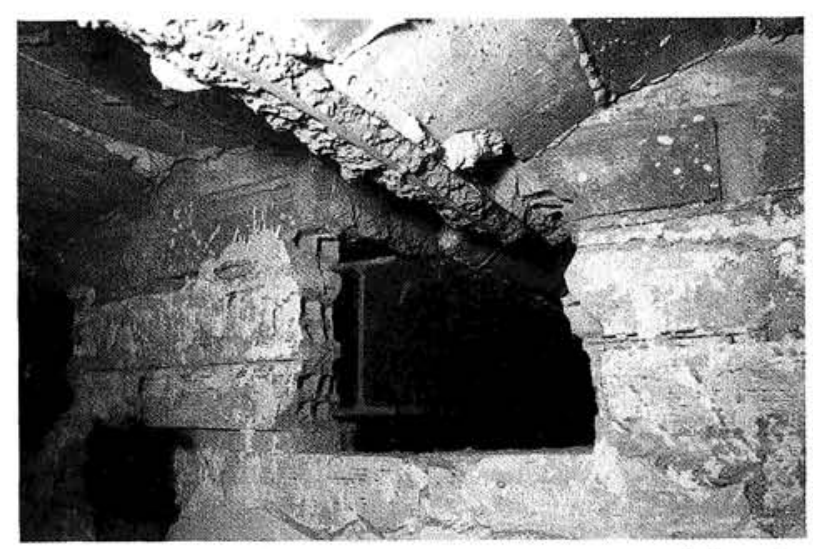

Foto 6.-El sistema de viga bajo viga parece que sigue siendo la mejor solución también en las viguetas fabricadas con cemento aluminoso.

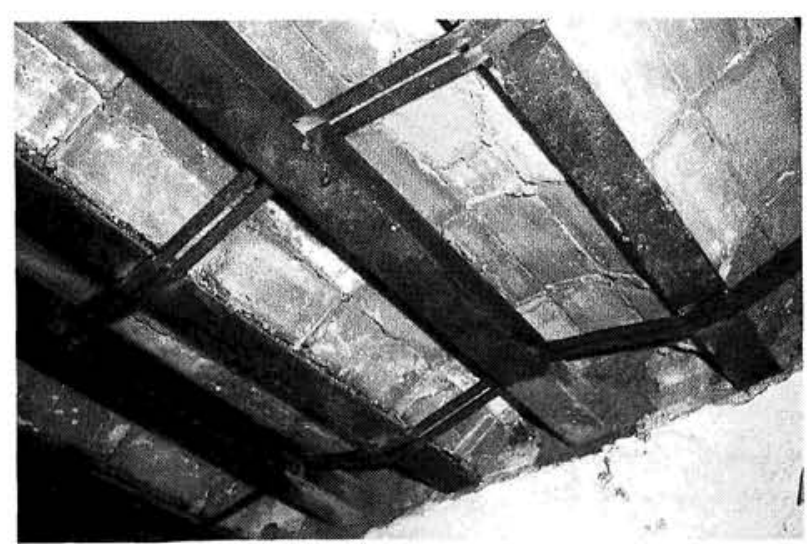

Foto 7.-La adaptación del sistema de viga bajo viga por problemas de altura libre, se puede resolver de diferentes formas.

(C) Consejo Superior de Investigaciones Científicas

Licencia Creative Commons 3.0 España (by-nc)

\section{SUBSTITUCIÓN FÍSICA Y FUNCIONAL}

Cuando nos encontramos ante un forjado que no ofrece garantías de seguridad estructural, o bien, cuando la durabilidad de sus componentes no se puede asegurar por un período prudencial, se plantea la necesidad de la substitución.

La substitución física resulta una intervención muy imporante dado que comporta la destrucción de elementos de tabiquería, pavimentación, acabados e instalaciones, que en muchos casos están en buen estado.

Para evitar el coste excesivo de estas intervenciones habitualmente se plantea substituir la función resistente del forjado sin destruirlo, a partir de la construcción de una estructura paralela a la existente capaz de soportar la totalidad de las cargas.

\section{Substitución física}

Posiblemente una de las mejores formas de eliminar la incertidumbre estructural que puede presentar un forjado en mal estado, es su substitución física por uno nuevo (foto 5).

Son muchos los condicionantes de tipo técnico y económico que convierten esta opción en una de las menos utilizadas. Actualmente se utiliza tan sólo en substituciones de algunas viguetas de forma individual o en pequeñas superficies de forjados en mal estado.

\section{Substitución funcional}

Los sistemas de substitución funcional que hoy por hoy se están utilizando se basan en colocar una viga bajo cada vigueta, vigas nuevas a cada lado de la vigueta existente y elementos pasadores, o bien la substitución funcional de un forjado en su totalidad mediante la construcción de otro nuevo adosado inferiormente al existente. 
Existen múltiples propuestas que intentan racionalizar estas actuaciones, haciéndolas más fáciles, rápidas y económicas. En todas ellas se adoptan sistemas autoportantes que transmiten la carga a la estructura de manera muy similar a la del forjado existente (fotos 6 y 7 ).

Quizás la variable más significativa en el diseño de una solución determinada es la elección del material de la nueva estructura:

- El acero laminado es un material que conjuga un buen comportamiento resistente con unas deformaciones fácilmente admisibles y una importante facilidad de manipulación (soldadura, corte, tornillería, etc.).

- La perfilería de acero conformado en frío (galvanizado, inoxidable, etc.) representa una alternativa que comporta más adaptabilidad para el diseño de determinadas geometrías y menor necesidad de protección (foto 8).

- El aluminio, se ha presentado también como alternativa. Es un material de baja densidad pero de bajo módulo elástico. Presenta una buena durabilidad si bien las soldaduras resultan problemáticas (foto 9).

- El hormigón prefabricado puede ser una solución para determinados casos, si bien presenta dificultades importantes para su colocación en obra a causa de su peso y difícil adaptabilidad (foto 10).

- Las estructuras tubulares en forma de celosía permiten, con un peso reducido y gran adaptabilidad, afrontar las cargas de un forjado, pero en general comportarán pérdidas de altura importanes y precauciones de protección y mantenimiento.

Utilizando los materiales comentados, se han estudiado diseños para adaptarlos a la complejidad de los trabajos de reparación. Hoy existen en el mercado sistemas comercializados (extensibles, telescópicos, desmontables, adaptables, etc.) que tienden hacia una facilidad de manipulación y colocación, evitando así lastimar los elementos existentes (tabiquerías, instalaciones, alicatados, muebles, etc.) (foto 11).

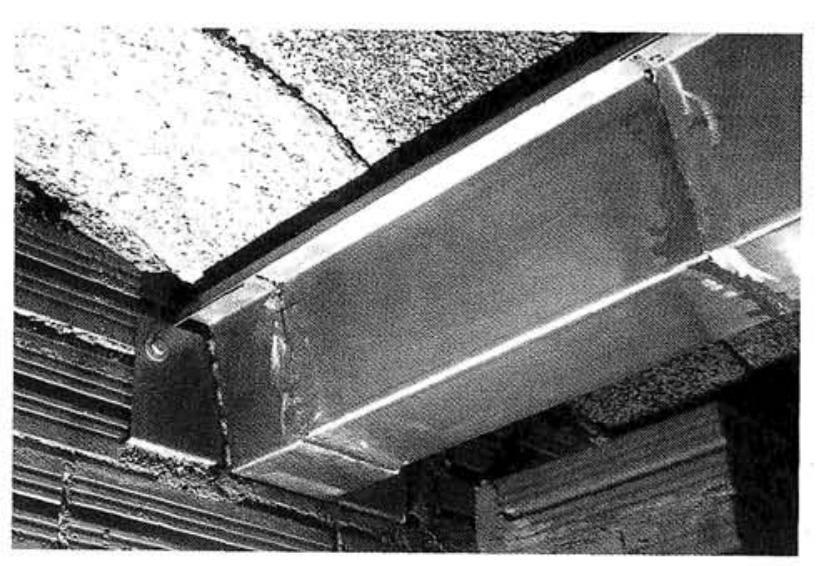

Foto 8.-La introducción de soluciones nuevas comercializadas puede resultar muy interesante si facilita el trabajo y se puede aplicar a precios competitivos.

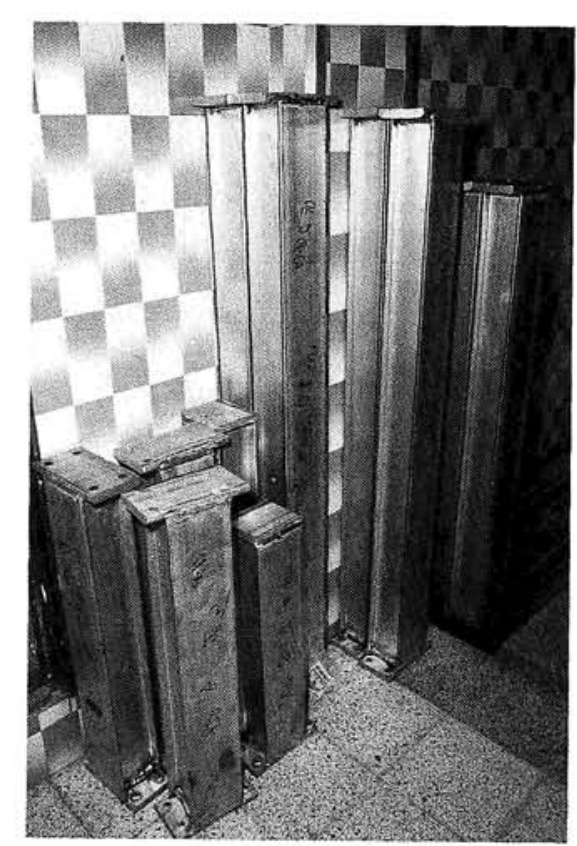

Foto 9.-Las piezas desmontables en éste u otro material resultan necesarias por su facilidad de transporte, manipulación y colocación.

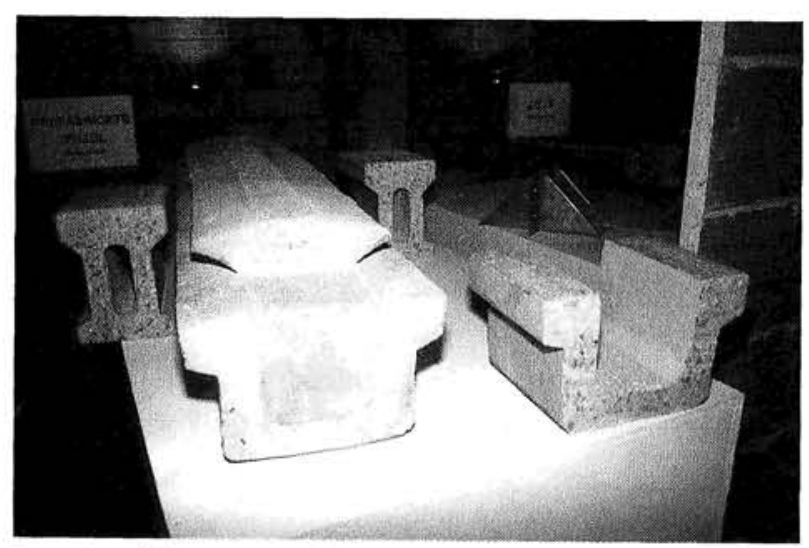

Foto 10.-El hormigón resulta de difícil manipulación y adaptabilidad. 


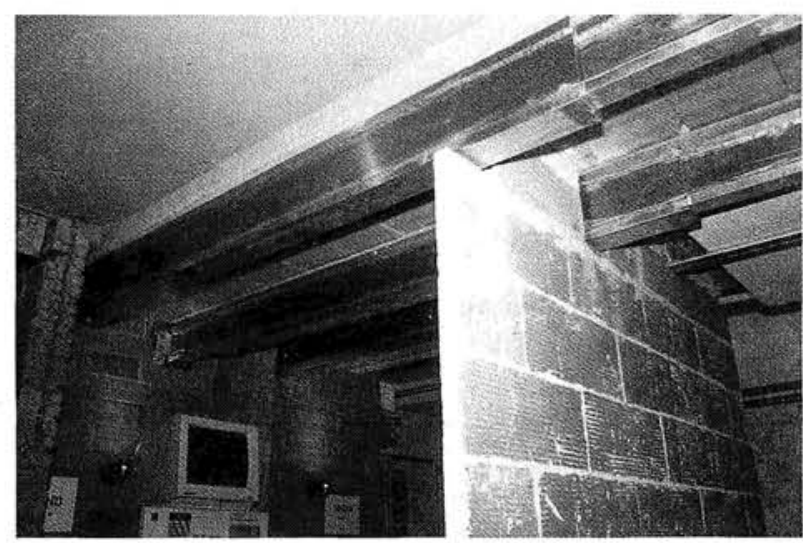

Foto 11.-Los sistemas telescópicos son también fáciles de transportar $y$ de montar.

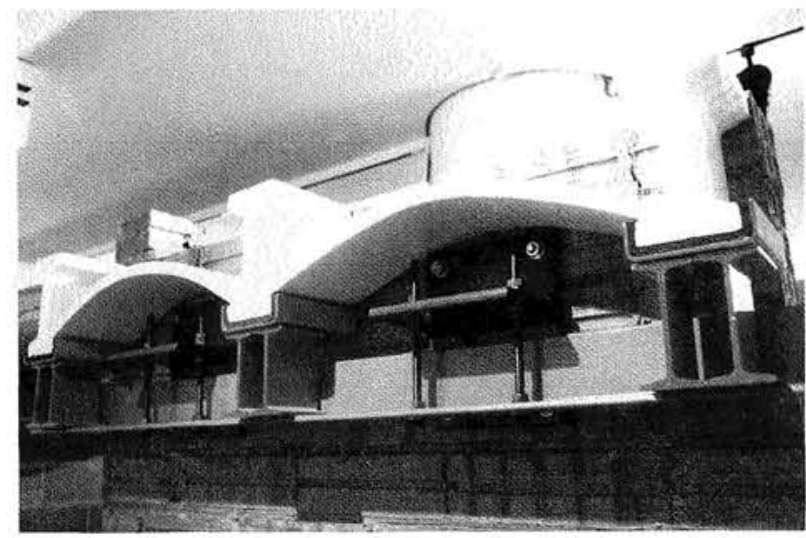

Foto 12.-Los apoyos son siempre puntos delicados en los que se nos presentan dificultades en puntos singulares. Hay que encontrar sistemas fácilmente adaptables y que ofrezcan una buena transmisión de esfuerzos.

Una problemática a considerar, que se presenta en casi todas las opciones, es el comportamiento al fuego de la solución adoptada, dado que los elementos metálicos y algunas resinas pueden presentar inconvenientes importantes.

Finalmente la discusión entre refuerzo activo y refuerzo pasivo permanece abierta.

Muchas de las soluciones comercializadas actúan inicialmente como simples "quitamiedos" y tan sólo soportarán cargas a partir de futuras deformaciones y en función de las rigideces respectivas.

Otras soluciones plantean ya inicialmente la puesta en carga del refuerzo en base a transmitir parte del esfuerzo soportado por la vigueta de hormigón al refuerzo, sin embargo la contraflecha a aplicar no está muy estudiada.

(c) Consejo Superior de Investigaciones Científicas

Licencia Creative Commons 3.0 España (by-nc)

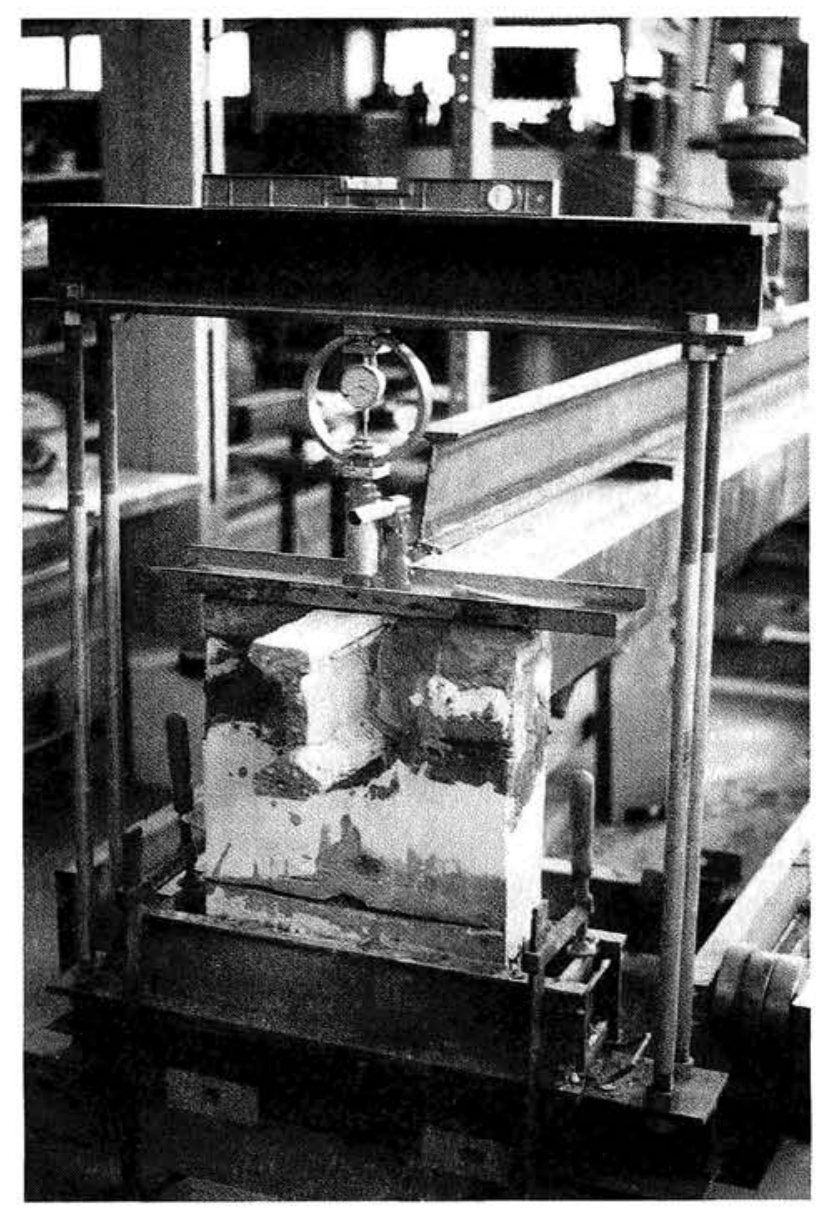

Foto 13.-Probar y contrastar las diferentes soluciones comerciales se hace imprescindible.

En cualquier caso, garantizar la homogeneidad de deformaciones es imprescindible.

\section{Sistemas de apoyo}

Uno de los puntos críticos que plantean estas actuaciones es la transmisión de los esfuerzos de los elementos horizontales a verticales, es decir, el punto de apoyo.

El tipo de actuación escogida, el tipo de forjado en el que se actúa, las características de los elementos portantes verticales y otros factores, contribuirán a hacer más sencilla o más dificil la transmisión de esfuerzos entre la estructura horizontal y la vertical, y a definir el sistema de apoyo idóneo a cada caso (foto 12).

Como criterios generales se debe procurar:

- No transmitir momentos a los muros.

http://informesdelaconstruccion.revistas.csic.es 
- Tener capacidad para absorber los cortantes.

- Repartir homogéneamente las cargas al soporte.

- Facilitar las labores de montaje.

- No producir una modificación importante del comportamiento estructural del edificio.

- Adaptabilidad a problemas singulares que se presenten en el proceso constructivo.

- Compatibilidad entre materiales.

\section{CONCLUSIONES}

Como se desprende del texto anterior, creemos que no existe una solución única a los problemas que aquí se han planteado, sino más concretamente unas filosofías de intervención. La opción a adoptar en cada caso concreto vendrá determinada, en cuanto se refiere a la inclusión en uno de los tres grupos de soluciones propuestas, por el resultado del proceso de diagnosis previo, $y$ en lo que se refiere a la solución concreta, en base a las características constructivas del edificio y muchos otros factores que hacen de cada caso un problema particular a estudiar en todos sus aspectos.

En cualquier caso, en este momento, parece descartada la solución por la vía química. Los especialistas en este campo tuvieron la posibilidad de incidir en el problema algunos años atrás, pero hoy por hoy, las soluciones, desde la óptica estricta de la seguridad de los edificios, son soluciones constructivas que se ven reforzadas en el proceso de diagnosis con sistemas analíticos que permiten aumentar la fiabilidad en la determinación de los períodos de vida útil.

Ya en la introducción al presente artículo, se ha planteado la necesidad de que hay que disponder de un marco unitario que avance en el estudio de este problema en todos sus aspectos. Concretamente en el tema de las soluciones se ve necesario establecer unos parámetros mínimos a cumplir en cada caso y exigir algún tipo de homologación de las propuestas comercializadas (foto 13) que garantice, al técnico que las aplique en un edificio, la bondad del sistema en sí mismo para así evitar que dentro de unos años nos encontremos nuevamente en una situación parecida a la actual por problemas de durabilidad o de comportamiento, en el tiempo, de soluciones que introducen nuevos materiales y sistemas no suficientemente contrastados.

Próxima aparición del libro:

\section{JORNADAS SOBRE CEMENTO ALUMINOSO}

Publicación sobre las Jornadas que tuvieron lugar en el ICCET, durante los días 4 y 5 de junio de 1992, con la colaboración del Instituto Español del Cemento y sus Aplicaciones (IECA).

En este libro se recogen todas las intervenciones manifestadas, así como las conferencias impartidas.

El número de ejemplares, será limitado y se podrán adquirir en el Instituto Eduardo Torroja. 